\title{
Evidence for Coexistence of Superconductivity and Magnetism in Single Crystals of Co-doped $\mathrm{SrFe}_{2} \mathrm{As}_{2}$
}

\author{
Jun Sung Kim, Seunghyun Khim, Liqin Yan, N. Manivannan, \\ Yong Liu, Ingyu Kim, G. R. Stewart ${ }^{\dagger}$, and Kee Hoon Kim* \\ FPRD, Department of Physics and Astronomy, Seoul National University, Seoul \\ 151-747, Republic of Korea \\ E-mail: khkim@phya.snu.ac.kr
}

\begin{abstract}
In order to investigate whether magnetism and superconductivity coexist in Co-doped $\mathrm{SrFe}_{2} \mathrm{As}_{2}$, we have prepared single crystals of $\mathrm{SrFe}_{2-x} \mathrm{Co}_{x} \mathrm{As}_{2}, x=0$ and 0.4 , and characterized them via $\mathrm{X}$-ray diffraction, electrical resistivity in zero and applied field up to $9 \mathrm{~T}$ as well as at ambient and applied pressure up to $1.6 \mathrm{GPa}$, and magnetic susceptibility. At $x=0.4$, there is both magnetic and resistive evidence for a spin density wave transition at $120 \mathrm{~K}$, while $T_{c}=19.5 \mathrm{~K}$ - indicating coexistent magnetism and superconductivity. A discussion of how these results compare with reported results, both in $\mathrm{SrFe}_{2-x} \mathrm{Co}_{x} \mathrm{As}_{2}$ and in other doped 122 compounds, is given.
\end{abstract}

PACS numbers: 74.70.Dd, 74.25.Ha, 74.25.Fy, 74.62.Fj 


\section{Introduction}

The recent discoveries of ever-mounting transition temperatures in the superconducting iron arsenside 2-dimensional layered compounds, coupled with the goal of understanding the pairing mechanism(s) of this newly discovered class of superconducting compounds, has lead to a surge of activity in materials-based condensed matter physics. ¿From a superconducting transition temperature $T_{c}=26 \mathrm{~K}$ in $\operatorname{LaFeAs}\left(\mathrm{O}_{1-x} \mathrm{~F}_{x}\right)$ [1] the value is now up to $T_{c}=55 \mathrm{~K}$ in $\operatorname{SmFeAs}\left(\mathrm{O}_{1-x} \mathrm{~F}_{x}\right)$ [2]. Of particular help in the quest for understanding this new physics has been the widening range of compounds in which the "iron arsenide (FeAs)" based superconductivity has been found, moving from the rather difficult materials synthesis of the original 1111 compounds with F-doping to the more-easily-prepared 122 compounds (non-superconducting prototype $\mathrm{BaFe}_{2} \mathrm{As}_{2}$ ) discovered by Rotter et al. [3] These latter compounds, as was pointed out by $\mathrm{Ni}$ et al. 4] can be rather easily grown from a Sn flux as well as from an FeAs 'self flux' [5]. Thus, much of the recent effort for elucidating the physics has focused on these 122 compounds, with both polycrystalline and single crystal work. Single crystals of course allow greater homogeneity and the possibility of following the anisotropy of the fundamental properties - often important in distinguishing the underlying mechanisms of superconductivity[6].

A central question[7] for deciding on the superconducting pairing mechanism in these FeAs superconductors has been the interplay/relationship between the ubiquitous magnetic behavior in the undoped, non-superconducting starting compounds (either the 1111 family or the $A \mathrm{Fe} 2 \mathrm{As} 2$, where $A=\mathrm{Ca}, \mathrm{Sr}, \mathrm{Ba}$, and $\mathrm{Eu}$ ) which is then suppressed by the doping. In the 122 family, $\mathrm{K} / \mathrm{Na} / \mathrm{Cs}$, or hole doping, on the $A$-site or $\mathrm{Co} / \mathrm{Ni}$ electron doping [5] - on the Fe site) induces superconductivity. Whether the magnetic

(spin density wave, 'SDW') behavior is coupled to the occurrence of superconductivity in $\mathrm{SrFe}_{2} \mathrm{As}_{2}$ doped with $\mathrm{Co}$ is a main subject of the present work, using single crystals prepared in Sn flux.

The question "does the SDW coexist with superconductivity in FeAs superconductors?" might seem straightforward to answer. However, even in just the 122 compounds, there exist at present four starting compounds $A \mathrm{Fe}_{2} \mathrm{As}_{2}(A=\mathrm{Ca}, \mathrm{Sr}, \mathrm{Ba}$, and Eu) with both hole (including work on $\mathrm{Na}, \mathrm{K}$ and $\mathrm{Cs}$ ) and electron (Co and $\mathrm{Ni}$ ) doping, and as well the very important materials aspects of both single- and poly-crystalline samples. Even a cursory review of the current status of this 4 ( Ca, Sr, Ba, Eu) $\times 2$ (hole/electron) $\times 2$ (single/poly) 'phase space' for just the 122 compounds already reveals both large differences but also serious conflicts between the various results. The rate at which doping depresses $T_{S D W}$ and induces superconductivity varies widely between the various $A$ atoms and either hole- or electron-dopants, which is a sign of the richness of this new class of materials. However, there are also conflicts in some results on the same $A$ atom and the same dopant which involve disagreements in concentration dependence of, e. g., $T_{S D W}$, in whether the SDW transition is first or second order in, $e$. g., $\mathrm{SrFe}_{2} \mathrm{As}_{2}$ [8, 9, 10, 11], and even in the quite fundamental question of coexistence of 
magnetism and superconductivity itself (see Table 1). Our work on the electron doped $\mathrm{SrFe}_{2} \mathrm{As}_{2}$ is the first to be done on single crystals in this compound (with one report on polycrystalline samples[12] and one on thin films[13]), bringing an initial data set for the $4 \times 2 \times 2$ set closer to completion. $\mathrm{SrFe}_{2} \mathrm{As}_{2}$ single crystals show a structural phase transition from a high-temperature tetragonal phase to a low-temperature orthorhombic phase at the same temperature as the SDW, $T_{o}=198 \mathrm{~K}$ [11] similar to the behavior observed in the $\mathrm{BaFe}_{2} \mathrm{As}_{2}$ compound [4].

As summarized in Table 1, the relation between magnetic behavior and superconductivity in the 122 FeAs superconductors has been addressed quite thoroughly for $A=\mathrm{Ba}$, but somewhat less so for $A=\mathrm{Ca}, \mathrm{Sr}$, and Eu. There is also growing work on electron doping (primarily Co replacing Fe) for all the $A$ species listed. As detailed in Table 1, at present the question of whether magnetism in the form of a SDW coexists with superconductivity in doped $A \mathrm{Fe}_{2} \mathrm{As}_{2}$ is still controversial.

Some of the disagreement in resolving the issue of coexistence of magnetism and superconductivity in the doped $122 \mathrm{AFe}_{2} \mathrm{As}_{2}$ materials made apparent by the summary in Table 1 can be resolved as merely based on interpretation. For example, some authors (e. g. see Refs. [17, 18]) have stated that the SDW transition is suppressed based on the lack of sharp structure in $\rho$ vs $T$ data, although a shoulder that might be indicative of a weak transition exists in their data. However, some of the disagreements appear to be fundamentally unresolvable at this time. One example of this involves contrasting $T_{S D W}$ vs $x$ results even in high quality single crystals of $\mathrm{BaFe}_{2-x} \mathrm{Co}_{x} \mathrm{As}_{2}$ by X. F. Wang et al. [21] and by J.-H. Chu et al. 22. Such disagreement is independent of any interpretation.

Two important lessons to be drawn from the summary in Table 1 on single crystal $\mathrm{SrFe}_{2-x} \mathrm{Co}_{x} \mathrm{As}_{2}$ are: (1) a fine gradation in composition in $\mathrm{BaFe}_{2-x} \mathrm{Co}_{x} \mathrm{As}_{2}$ was shown to be necessary for determining whether $T_{S D W}$ has been suppressed to $T=0$ when superconductivity first appears[21, 22, 23] (2) Some of the work on polycrystalline samples has been found to disagree with single crystal work, partly at least for reasons still under discussion, thus obscuring any possible conclusions. In general, although single crystals grown in Sn-flux can have small inclusions of $\mathrm{Sn} 4$, single crystals should be more homogeneous than sintered polycrystalline material. In order to address point (1), we are working on single crystals of $\mathrm{SrFe}_{2-x} \mathrm{Co}_{x} \mathrm{As}_{2}, x=0.1,0.2,0.3$, and 0.5 in addition to the work on $x=0$ and 0.4 reported here. However, as will be discussed below, $T_{S D W}$ is suppressed much less rapidly with $\mathrm{Co}$ in $\mathrm{SrFe}_{2-x} \mathrm{Co}_{x} \mathrm{As}_{2}$ than in $\mathrm{BaFe}_{2-x} \mathrm{Co}_{x} \mathrm{As}_{2}$, and our present work on $x=0$ and 0.4 is sufficient to show the coexistence of magnetism and superconductivity in $\mathrm{SrFe}_{2-x} \mathrm{Co}_{x} \mathrm{As}_{2}$ - contradicting conclusions based on polycrystalline $\mathrm{SrFe}_{2-x} \mathrm{Co}_{x} \mathrm{As}_{2}[12$ (see Table 1).

\section{Experimental}

Single crystals of Co-doped $\mathrm{SrFe}_{2} \mathrm{As}_{2}$ were grown using high temperature solution growth techniques with a Sn flux[4]. Stoichiometric amounts of the elemental Sr, Fe, Co and As were added to $\mathrm{Sn}$ with the ratio of $\left[\mathrm{SrFe}_{2-x} \mathrm{Co}_{x} \mathrm{As}_{2}\right]: \mathrm{Sn}=1: 20$ and placed in an 
Table 1. Survey of previous doping results in 122 FeAs superconductors. Units of temperature are Kelvin; results are for either single- or poly-crystalline samples. It is worth noting that some authors, well-focused on the difficulty of answering the coexistence question precisely, have used more precise determination of $T_{S D W}(e . g$., Wang et al.[21] used specific heat; Zhang et al.[16] used band splitting measured by photoemission).

\begin{tabular}{lllllll}
\hline $\begin{array}{l}A_{1-x} A_{x}^{\prime} \mathrm{Fe}_{2-y} \mathrm{Co}_{y} \mathrm{As}_{2} \\
\left(A^{\prime}=\mathrm{K}, \mathrm{Na}\right)\end{array}$ & Dopant $_{x, y}$ & $T_{S D W}$ & $T_{c}$ & $\begin{array}{l}\text { Coexistence } \\
\text { (yes/no) }\end{array}$ & $\begin{array}{l}\text { single } \\
\text { /poly }\end{array}$ & {$[\mathrm{Ref}]$.} \\
\hline$A=\mathrm{Ca}$ & $\mathrm{Co}_{0.06}$ & none & 17 & no & single & {$[14]$} \\
& $\mathrm{Na}_{0.5}$ & none & 20 & no & poly & {$[15]$} \\
\hline$A=\mathrm{Sr}$ & $\mathrm{K}_{0.2}$ & 135 & 25 & yes & single & {$[16]$} \\
& $\mathrm{K}_{0.4}$ & none & 38 & no & single & {$[17]$} \\
& $\mathrm{K}_{0.4}$ & none & $20^{*}$ & no & poly & {$[18]$} \\
& $\mathrm{Na}_{0.5}$ & 160 & 35 & yes & single & {$[19]$} \\
& $\mathrm{Co}_{0.2}$ & none & 19.2 & no & poly & {$[12]$} \\
\hline$A=\mathrm{Ba}$ & $\mathrm{K}_{0.5}$ & 70 & 37 & yes & single & {$[19]$} \\
& $\mathrm{K}_{0.2,0.3}$ & 120,100 & 7,14 & yes & poly & {$[20]$} \\
& $\mathrm{Co}_{0.17}$ & 75 & 9 & yes & single & {$[21]$} \\
& $\mathrm{Co}_{0.10}$ & $35-50($ split $)$ & 20 & yes & single & {$[22]$} \\
& $\mathrm{Co}_{0.16}$ & none & 22 & no & single & {$[5,23]$} \\
\hline$A=\mathrm{Eu}$ & $\mathrm{K}_{0.5}$ & none & 32 & no & poly & {$[24]$} \\
& Pressure & 115 & 30 & yes & single & {$[25]$} \\
\hline
\end{tabular}

* Annealed polycrystalline $\mathrm{Sr}_{0.6} \mathrm{~K}_{0.4} \mathrm{Fe}_{2} \mathrm{As}_{2}$ changes $T_{c}$ from 38 to $20 \mathrm{~K}$. In the unannealed state, there is an anomaly in $\rho$ at $200 \mathrm{~K}$ indicative of SDW and $T_{c}=$ $38 \mathrm{~K}[18$.

alumina crucible, which was sealed in a silica ampoule in vacuum. All the handling of the elements was performed in a glove box with an Ar atmosphere (oxygen $<1 \mathrm{ppm}$, $\mathrm{H}_{2} \mathrm{O}<1 \mathrm{ppm}$ ). The sealed crucible was heated to $700{ }^{\circ} \mathrm{C}$ (duration of 4 hours), then to $1100{ }^{\circ} \mathrm{C}$ (duration of 4 hours). After this, the sample was slowly cooled down to 500 ${ }^{\circ} \mathrm{C}$ at the rate of $4{ }^{\circ} \mathrm{C} /$ hour and then the plate-like single crystals of typical dimensions $10 \times 10 \times 0.5 \mathrm{~mm}^{3}$ were removed from the Sn flux by centrifuging 4 .

Resistivity measurements were made by a standard 4-wire ac method, using a Quantum Design PPMS ${ }^{\mathrm{TM}}$ system in fields up to $9 \mathrm{~T}$. Due to the large flat faces of the crystals, where the c-axis is perpendicular to the face, alignment of the field either parallel to the c-axis or in the ab-plane was straightforward. Magnetic susceptibility measurements were performed in the same Quantum Design PPMS ${ }^{\mathrm{TM}}$ system.

\section{Results and discussion}

X-ray diffraction measurements were carried out on a single crystal from both of the compositions $x=0$ and 0.4. As shown in Fig. 1, only (00l) reflections with even $l$ appear, indicating that the $c$-axis is perpendicular to the crystal plate. The addition of Co decreased the $c$-axis lattice parameter in these single crystals. However, for reasons 


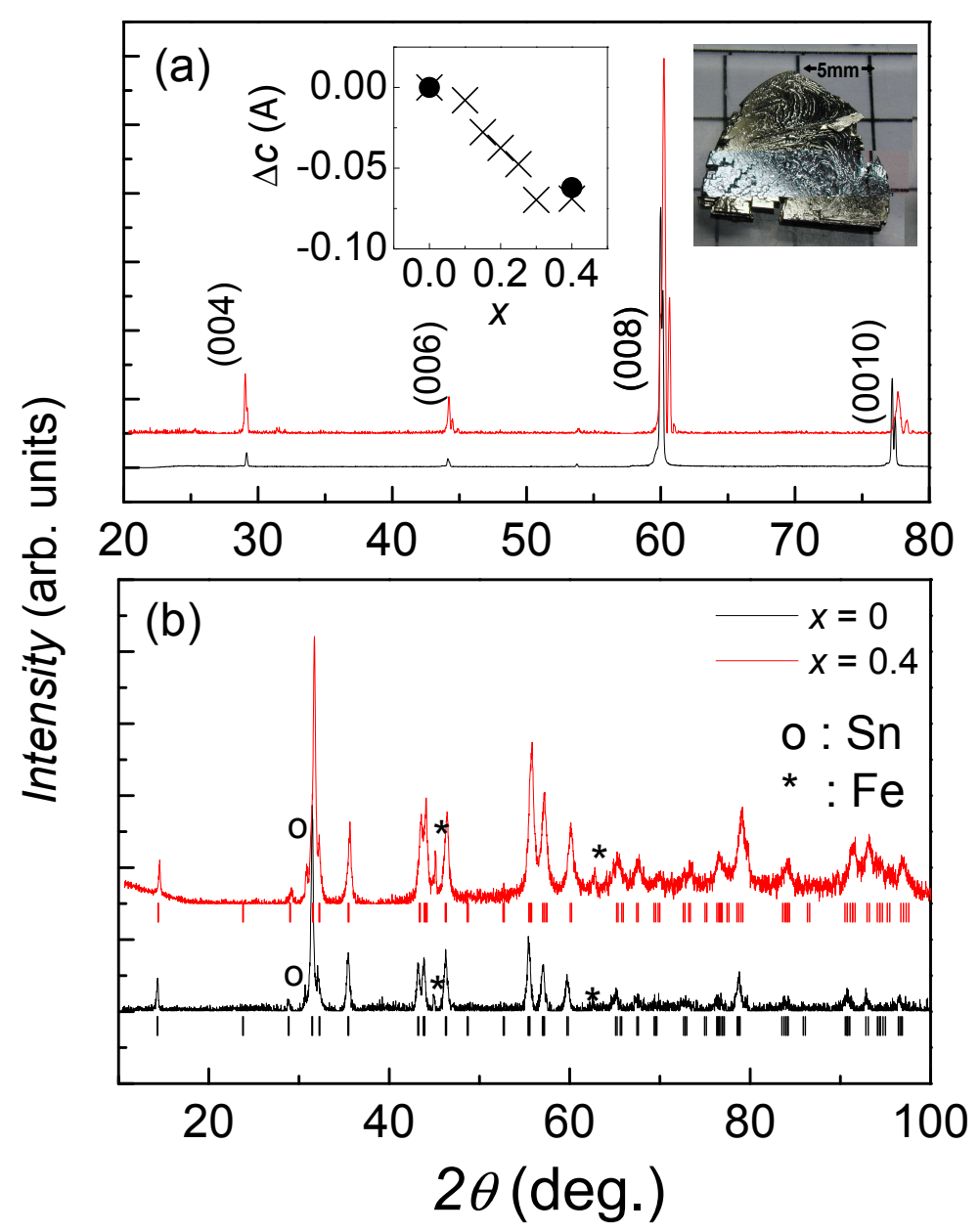

Figure 1. (Color online) X-ray diffraction patterns for (a) single crystal and (b) crushed powder of $\mathrm{SrFe}_{2-x} \mathrm{Co}_{x} \mathrm{As}_{2}$ with $x=0$ and 0.4. The upper left inset shows the decrease of the $c$-axis lattice constant $(\Delta c)$ in the single crystals of the present work due to Co doping (solid circle). For comparison, we also plot the $\Delta c$ vs $x$ for polycrystalline $\mathrm{SrFe}_{2-x} \mathrm{Co}_{x} \mathrm{As}_{2}$ (× symbols) [12. The upper middle inset shows a photo of a $x=0.4$ single crystal. Note the marked second phase lines in the powder pattern (b), where in addition to a slight amount of Sn inclusion from the flux, some excess Fe is seen.

that will become clear below in the discussion of the resistivity data for $x=0.4$, in order to investigate possible crystal inhomogeneity and impurities below the rather shallow penetration of the X-ray beam $(\sim$ a few $\mu \mathrm{m})$ in the single crystals, we undertook X-ray diffraction of powders made of individual single crystals. These data, shown also in Fig. 1 , provide a measurement of both the $a$ - and $c$-axis lattice parameters and are more characteristic of the bulk of the crystal. These powder diffraction lattice parameter results agree with the single crystal results. The results for $x=0$ were $a=b=3.928(3)$ $\AA, c=12.392(9) \AA$, while $a=b=3.925(3) \AA, c=12.33(1) \AA$ for $x=0.4$. For $x$ $=0$, the $c$-axis lattice parameter is consistent with some previous reports on poly- 8 ] and single-crystal materials [17, but is slightly larger than the polycrystalline results of Leithe-Jasper et al.[12] With Co doping, it has been shown that the c-axis lattice 


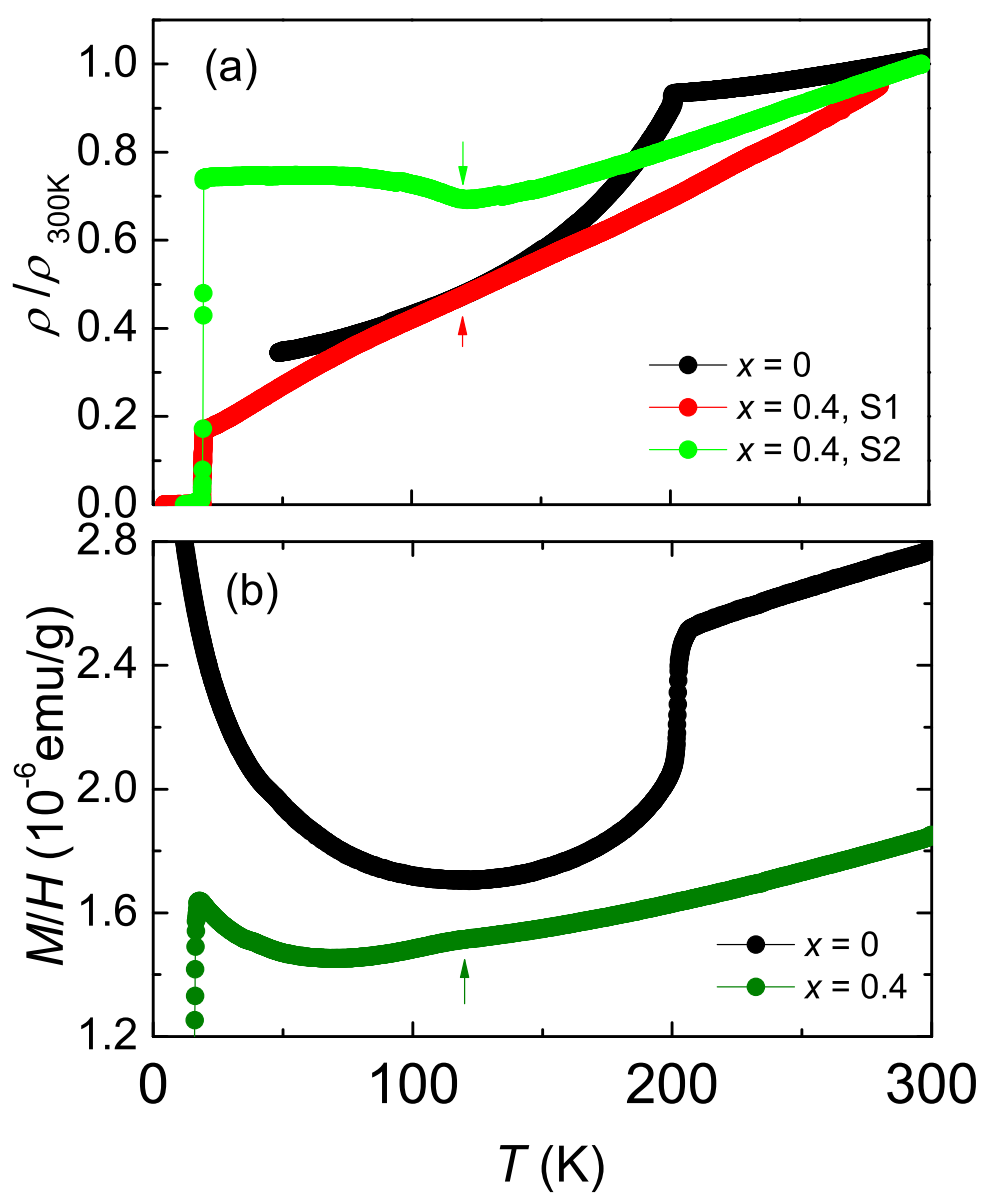

Figure 2. (Color online) Resistivity vs temperature (a) for single crystal $\mathrm{SrFe}_{2-x} \mathrm{Co}_{x} \mathrm{As}_{2}, x=0$ and 0.4 (two samples from the same growth batch, labeled $\mathrm{S} 1$ and S2), showing the anomalies at $T_{S D W}(202 \mathrm{~K}$ for $x=0$ and $\approx 120 \mathrm{~K}$ for $x=$ 0.4). On an expanded plot, not shown, extrapolations of the higher temperature and separately the lower temperature resistivity data from the data for S1 intersect at the temperature marked by the red arrow. The plot in (b) shows the $M / H$ (measured in $7 \mathrm{~T}$ ), where the SDW anomalies for both $x=0$ and 0.4 are clear.

parameter decreases linearly with Co concentration[12, 21, 22].

Considering the inconsistency in the the absolute value of the $c$-axis lattice parameters in the literature, we focused here on a comparison of the change(contraction) of the $c$-axis in our Co-doped crystals with that found in polycrystalline works [12] (see Fig. 1). According to Leithe-Jasper et al. a $c$-axis contraction of $-0.07(1) \AA$ is expected for $x=0.4$, which is comparable with our result of -0.06(2) $\AA$ for our Co-doped single crystal. This provides a bulk proof for the presence of approximately $x=0.4$ Co in our Co-doped single crystals.

Resistivity and susceptibility data for $x=0$ and 0.4 are shown in Figure 2. Discussing the normal state properties first, as shown clearly in Fig. 2, our single crystals for $x=0.4$ show differing resistivity behaviors below $T_{S D W}$ : one crystal (S2) 
shows evidence for strong scattering below $T_{S D W}$ while another crystal (S1) shows only a slight change in slope (marked by the red arrow). [We have measured a total of 6 different single crystals out of the same growth batch for $x=0.4$, and the strong increase in $\rho$ below $T_{S D W}$ is found in two samples. We are continuing to investigate this.] This sample dependence is of course reminiscent of early sample dependence problems in $\rho$ in $\mathrm{YBa}_{2} \mathrm{Cu}_{3} \mathrm{O}_{7-\delta}$. However, in both crystals (as well in all the other crystals measured from this $x=0.4$ batch), the superconducting transition is consistently at $T_{c}=19.5 \mathrm{~K}$. Clearly, the magnetic anomaly for $\mathrm{SrFe}_{1.6} \mathrm{Co}_{0.4} \mathrm{As}_{2}$ is a clearer evidence for a SDW transition at $120 \mathrm{~K}$ than the slight change of slope/broad hump in the resistivity data that is characteristic of most of our samples. In the polycrystalline work on $\mathrm{SrFe}_{2-x} \mathrm{Co}_{x} \mathrm{As}_{2}$ [12], the resistivity curve for a nonsuperconducting sample of $x=0.1$ increases below $T \sim 130 \mathrm{~K}$ similar to the S2 data for the $x=0.4$ single crystal in Fig. 2. The polycrystalline resistivity data[12 for $x \geq 0.2\left(T_{c}=19.2 \mathrm{~K}\right.$ for $\left.x=0.2\right)$ show positive curvature vs temperature between $T_{c}$ and $300 \mathrm{~K}$, i.e. unlike both the $\mathrm{S} 2$ and $\mathrm{S} 1$ resistivity curves for our single crystal $\mathrm{SrFe}_{2-x} \mathrm{Co}_{x} \mathrm{As}_{2}$ shown in Fig. 2. Thus, if it were not for the good agreement in the lattice contraction for the same compositions $(x=0$ and 0.4$)$ in the present single crystal work compared to the polycrystalline work [12], both the difference in the behavior of $T_{S D W}$ and $T_{c}$ would have called the comparability of the Co-compositions into question. As it is, it would be useful for magnetic susceptibility data to higher temperatures than $25 \mathrm{~K}$ (the upper limit in Ref. 12) to be measured on the polycrystalline samples. At this time it is not clear why there is disagreement between $T_{S D W}(x)$ results determined by resistivity data on poly-[12] and single-crystalline (present work) samples of $\mathrm{SrFe}_{2-x} \mathrm{Co}_{x} \mathrm{As}_{2}$.

As stated in the Introduction, the field of FeAs superconductivity is in a state of flux at present. The variation of the resistivity seen in our single crystals for $x=0.4$, and the disagreement between our single crystal compositional dependence of $T_{S D W}$ and $T_{c}$ compared to polycrystalline[12] results is perhaps one reason why some of these open questions must remain open until better understanding of sample quality is achieved.

The superconducting transition temperature for $x=0.4 \mathrm{SrFe}_{2-x} \mathrm{Co}_{x} \mathrm{As}_{2}$ single crystals is $19.5 \mathrm{~K}$, which is comparable with the maximum $T_{c}$ achieved by Co-doping in polycrystalline $\mathrm{SrFe}_{2} \mathrm{As}_{2}\left[12\right.$ and coexists with the magnetic transition at $T_{S D W} \approx 120$ K. Our work in progress on other compositions confirms this result, adding one more piece to the conclusion that is becoming clearer (see Table 1 and references therein) that - contrary to early conclusions, magnetism and superconductivity clearly coexist in these $122 \mathrm{FeAs}$ superconductors. In comparison with $\mathrm{Co}$-doped $\mathrm{BaFe}_{2} \mathrm{As}_{2}$, the magnetic phase is more robust in Co-doped $\mathrm{SrFe}_{2} \mathrm{As}_{2}$. For $\mathrm{BaFe}_{2} \mathrm{As}_{2}$, the $T_{S D W}$ is decreased rapidly with a relatively small amount of Co substitution, $i$. $\quad$ e., $x=0.12$, which is sufficient to fully suppress the SDW transition and induce the maximum $T_{c} \approx 24 \mathrm{~K}$. In contrast, we still observe the clear magnetic transition at $T_{S D W} \approx 120 \mathrm{~K}$ with $x=0.4$ in $\mathrm{SrFe}_{2-x} \mathrm{Co}_{x} \mathrm{As}_{2}$ with $T_{c} \approx 20 \mathrm{~K}$. This result may be related to the higher $T_{S D W} \approx$ $202 \mathrm{~K}$ in $\mathrm{SrFe}_{2} \mathrm{As}_{2}$ than that of $\mathrm{BaFe}_{2} \mathrm{As}_{2}\left(T_{S D W} \approx 140 \mathrm{~K}\right)$.

The temperature-dependence of $H_{c 2}(T)$, defined by $90 \%$ of the resistive transition 


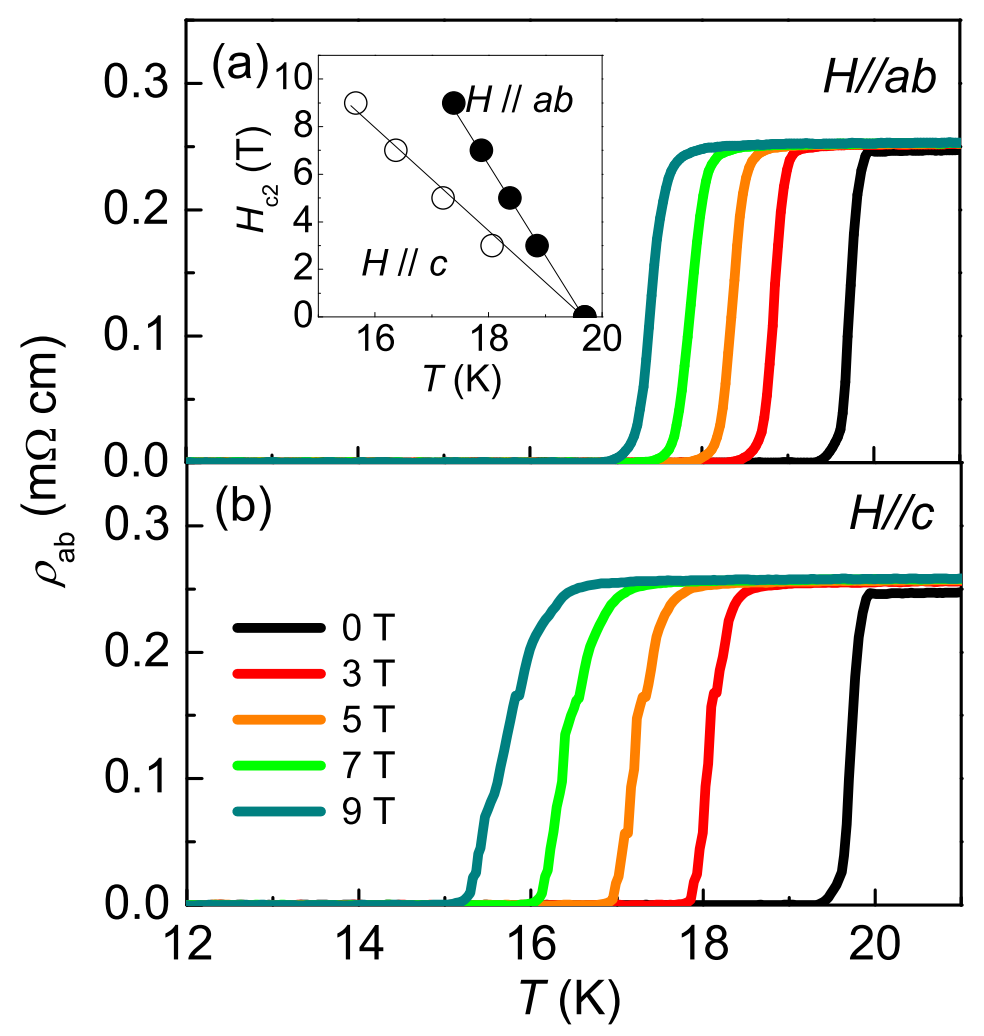

Figure 3. (Color online) Temperature dependence of the $a b$-plane resistivity of single crystal $\mathrm{SrFe}_{1.6} \mathrm{Co}_{0.4} \mathrm{As}_{2}$ with different magnetic fields along the $a b$-plane (a) and the $c$-axis (b). The inset shows the $H_{c 2}(T)$ curves near $T_{c}$ for the two field directions, $H$ $\| c$ and $H \| a b$.

is shown in the inset of Fig. 3. Both $H_{c 2}^{a b}$ and $H_{c 2}^{c}$ showed almost linear temperature dependence with slopes of $d H_{c 2}^{a b} / d T=-3.9 \mathrm{~T} / \mathrm{K}$ and $d H_{c 2}^{c} / d T=-2.2 \mathrm{~T} / \mathrm{K}$. The zero temperature upper critical fields can be estimated using the Werthamer-HelfandHohenberg formula, $H_{c 2}(0)=-\left.0.69 T_{c}\left(d H_{c 2} / d T\right)\right|_{T_{c}}$, yielding $H_{c 2}^{c}(0)=30 \mathrm{~T}$ and $H_{c 2}^{a b}(0)$ $=53 \mathrm{~T}$. The corresponding coherence lengths are $33 \AA$ and $19 \AA$ along the $a b$-plane and the $c$-axis, respectively. The $c$-axis coherence length is comparable with the distance between two adjacent FeAs layers, $d \sim 6 \AA$, indicating the quasi- 2 dimensionality of the superconductivity. The anisotropy parameter $\Gamma=H_{c 2}^{a b} / H_{c 2}^{c}$ derived from the data in Fig. 3 is $\Gamma \approx 1.7$, which is comparable with $\Gamma \approx 1.5-2$ of $\mathrm{K}$ - or Co-doped $\mathrm{BaFe}_{2} \mathrm{As}_{2}$ and $\mathrm{K}$-doped $\mathrm{SrFe}_{2} \mathrm{As}_{2}$ [17] but significantly lower than $\Gamma \approx 5-10$ in the 1111 oxypnictides.

As a final characterization of the superconductivity we observe in our single crystals of $\mathrm{SrFe}_{1.6} \mathrm{Co}_{0.4} \mathrm{As}_{2}$, we present the pressure dependence of $T_{c}$ in Fig. 4. Gooch et al. [26] reported on $T_{c}(P)$ in polycrystalline $\mathrm{Sr}_{0.6} \mathrm{~K}_{0.4} \mathrm{Fe}_{2} \mathrm{As}_{2}, T_{c}^{\text {onset }}=37 \mathrm{~K}$, and find an increase in $T_{c}^{\text {onset }}$ at $0.9 \mathrm{GPa}$ of about $1.2 \mathrm{~K}$, or about $3 \%$, compared to our result for electrondoped $\mathrm{SrFe}_{2} \mathrm{As}_{2}$ where $T_{c}$ increases by about $1.8 \mathrm{~K}$, or about $9 \%$ with $0.9 \mathrm{GPa}$. Gooch et al. also see some saturation in the rise of $T_{c}$ with pressure in their $1.7 \mathrm{GPa}$ data comparable to what we observe (see inset to Fig. 4). From previous pressure work on 


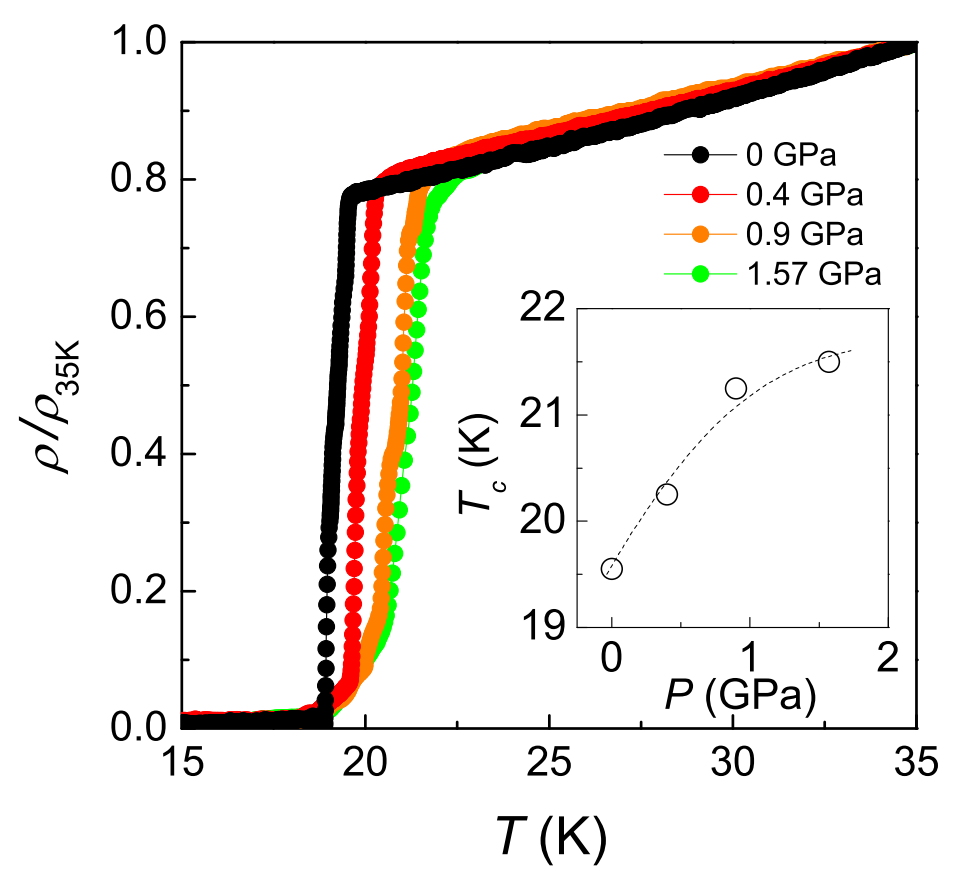

Figure 4. (Color online) Superconducting transition temperatures for single crystal $\mathrm{SrFe}_{1.6} \mathrm{Co}_{0.4} \mathrm{As}_{2}$ determined by the resistivity as a function of hydrostatic pressure

the $\mathrm{K}$-doped $A \mathrm{Fe}_{2} \mathrm{As}_{2}(A=\mathrm{Ba}, \mathrm{Sr})$ compounds, it has been found that the pressure dependence of $T_{c}$ reflects the "dome" shape of the doping dependence of $T_{c}[26$. The underdoped and overdoped samples show positive and negative pressure dependence, respectively, while almost no pressure dependence of $T_{c}$ is observed in the optimally doped sample. As mentioned already, our $x=0.4$ crystal shows $T_{c}=19.5 \mathrm{~K}$, close to the maximum $T_{c}$ of Co-doped polycrystalline $\mathrm{SrFe}_{2} \mathrm{As}_{2}[12$, thus in the optimal doping regime. The sizable pressure dependence of $T_{c}$ in our crystal, therefore, suggests that there is still room for improving the superconducting transition temperature by further tuning, e. g., using external pressure. Similar behavior has been also observed in optimally Co-doped $\mathrm{BaFe}_{2} \mathrm{As}_{2}$ [27]. This different behavior between K-doped and Codoped 122 compounds indicates that the pressure dependence of $T_{c}$ is determined not just by the doping level of the FeAs layer but also reflects more complex interplay with other parameters such as the degree of hybridization between the Fe and As states that can be tuned by, e. g., a bonding angle of the Fe-As-Fe network[28].

\section{Summary and Conclusion}

Our present work on single crystal $\mathrm{SrFe}_{2-x} \mathrm{Co}_{x} \mathrm{As}_{2}(x=0$ and 0.4$)$ shows clear signatures in both electrical resistivity and magnetization curves for the presence of a spin density wave at 202 and $120 \mathrm{~K}$, respectively. The $x=0.4$ sample shows superconductivity at 19.5 K, which - in the spirit of the work on the FeAs superconductors to date (see Table 1) - allows the conclusion that superconductivity is coexistent with magnetism 
(SDW) in single crystal $\mathrm{SrFe}_{1.6} \mathrm{Co}_{0.4} \mathrm{As}_{2}$. Of course, a microscopic determination of the coexistence below $T_{c}$ is further required. Both the single crystal and powder Xray diffraction characterization of our samples show internal consistency as well as agreement of the lattice contraction with Co doping, compared to the polycrystalline work on $\mathrm{SrFe}_{2-x} \mathrm{Co}_{x} \mathrm{As}_{2}$ 12. In contrast, our compositional dependence of both $T_{S D W}$ and $T_{c}$ disagree with the polycrystalline data in Ref. 12 which does not report magnetic susceptibility. The anisotropy of the upper critical field $H_{c 2}$ in our single crystals of $\mathrm{SrFe}_{1.6} \mathrm{Co}_{0.4} \mathrm{As}_{2}$ is consistent with $\mathrm{K}$ - or Co-doped $\mathrm{BaFe}_{2} \mathrm{As}_{2}$ and $\mathrm{K}$-doped $\mathrm{SrFe}_{2} \mathrm{As}_{2}$ [17]. The pressure dependence of $T_{c}$ of our single crystalline $\mathrm{SrFe}_{1.6} \mathrm{Co}_{0.4} \mathrm{As}_{2}$ is, when expressed as a percentage of $T_{c}(P=0)$, much larger than that observed [26] in $\mathrm{K}$-doped $\mathrm{SrFe}_{2} \mathrm{As}_{2}$.

An important conclusion that can be drawn from our present work is that even in single crystals there appears to be significant sample dependence at least in the resistivity below $T_{S D W}$, while $T_{S D W}$ and $T_{c}$ themselves did not show any sample dependence. Our results clearly show sample dependence in the resistivity, as well as an unexplained difference between our single crystal and Ref. 12's polycrystalline values of $T_{S D W}$ and $T_{c}$ as a function of Co-concentration. This may be a useful cautionary note about the rush to make firm conclusions in the early stages of the fascinating study of superconductivity in the 122 FeAs compounds.

\section{Acknowledgments}

This work was supported by the National Research Lab program (M10600000238) and KICOS through a grant provided by the MEST (K20702020014-07E0200-01410). Work at Florida supported by the US Department of Energy, contract no. DE-FG0286ER45268. JSK is supported by BK21 Frontier Physics Research Division.

\section{References}

${ }^{*}$ Corresponding author.

†On sabbatical from Department of Physics, University of Florida.

[1] Kamihara Y, Watanabe T, Hirano M, and Hosono H 2008 J. Am. Chem. Soc. 1303296.

[2] Ren Z A, Wei L, Jie Y, Wei Y, Xiao-Li S, Zheng-Cai, Guang-Can C, Xiao-Li D, Li-Ling S, Fang Z and Zhong-Xian Z 2008 Chin. Phys. Lett. 252215.

[3] Rotter M, Tegel M, and Johrendt D 2008 Phys. Rev. Lett. 101107006.

[4] Ni N, Bud'ko S L, Kreyssig A, Nandi S, Rustan G E, Goldman A I, Gupta S, Corbett J D, Kracher A, and Canfield P C 2008 Phys. Rev. B 78014507.

[5] Sefat A S, Jin R, McGuire M A, Sales B C, Singh D J, and Mandrus D 2008 Phys. Rev. Lett. 101 117004 .

[6] Tsuei C C and Kirtley J R 2000 Rev. Mod. Phys. 72969.

[7] See, for example, M. J. Han et al. Priprint arXiv0811.0034 who state "Our result strongly suggests the magnetic fluctuation as the pairing mechanism for the superconducting ground state" in these Fe-based superconductors.

[8] Krellner C, Caroca-Canales N, Jesche A, Rosner H, Ormeci A, and Geibel C 2008 Phys. Rev. B 78 100504(R). 
[9] Chen G F, Li Z, Li G, Hu W Z, Dong J, Zhang X D, Zheng P, Wang N L, and Luo J L 2008 Chin. Phys. Lett. 253403.

[10] Tegel M, Rotter M, Weiss V, Schappacher F M, Poettgen R and Johrendt D Priprint arXiv0806.4782.

[11] Yan J -Q, Kreyssig A, Nandi S, Ni N, Bud'ko S L, Kracher A, McQueeney R J, McCallum R W, Lograsso T A, Goldman A I, Canfield P C 2008 Phys. Rev. B. 78024516.

[12] Leithe-Jasper A, Schnelle W, Geibel C, and Rosner H Priprint arXiv0807.2223.

[13] Hiramatsu H, Katase T, Kamiya T, Hirano M, and Hosono H 2008 Appl. Phys. Express 1101702.

[14] Kumar N, Nagalakshmi R, Kulkarni R, Paulose P L, Nigam A K, Dhar S K, Thamizhavel A Priprint arXiv0810.0848.

[15] Wu G, Chen H, Wu T, Xie Y L, Yan Y J, Liu R H, Wang X F, Ying J J, and Chen X H Priprint arXiv0806.4279.

[16] Zhang Y, Wei J, Ou H W, Zhao J F, Zhou B, Chen F, Xu M, He C, Wu G, Chen H, Arita M, Shimada K, Namatame H, Taniguchi M, Chen X H, Feng D L Priprint arXiv0808.2738.

[17] Chen G F, Li Z, Dong J, Li G, Hu W Z, Zhang X D, Song X H, Zheng P, Wang N L, and Luo J L 2008 Phys. Rev. B $\mathbf{7 8} 224512$.

[18] Chen G F, Li Z, Li G, Hu W Z, Dong J, Zhang X D, Zheng P, Wang N L, and Luo J L 2008 Chin. Phys. Lett. 253403.

[19] Goko T, Aczel A A, Baggio-Saitovitch E, Bud'ko S L, Canfield P C, Carlo J P, Chen G F, Dai P, Hamann A C, Hu W Z, Kageyama H, Luke G M, Luo J L, Nachumi B, Ni N, Reznik D, Sanchez-Candela D R, Savici A T, Sikes K J, Wang N L, Wiebe C R, Williams T J, Yamamoto T, Yu W, and Uemura Y J Priprint arXiv0808.1425.

[20] Chen H, Ren Y, Qiu Y, Bao W, Liu R H, Wu G, Wu T, Xie Y L, Wang X F, Huang Q, and Chen X H Priprint arXiv0807.3950.

[21] Wang X F, Wu T, Wu G, Liu R H, Chen H, Xie Y L and Chen X H Priprint arXiv0811.2920.

[22] Chu J-H, Analytis J G, Kucharczyk C, Fisher I R Priprint arXiv0811.2463.

[23] Ning F L, Ahilan K, Imai T, Sefat A S, Jin R, McGuire M A, Sales B C, Mandrus D Priprint arXiv0811.1617.

[24] Jeevan H S, Hossain Z, Kasinathan D, Rosner H, Geibel C, and Gegenwart P 2008 Phys. Rev. B 78, 092406.

[25] Miclea C F, Nicklas M, Jeevan H S, Kasinathan D, Hossain Z, Rosner H, Gegenwart P, Geibel C, and Steglich F Priprint arXiv0808.2026.

[26] Gooch M, Lv B, Lorenz B, Guloy A M, and Chu C-W 2008 Phys. Rev. B 78 180508(R).

[27] Ahilan K, Balasubramaniam J, Ning F L, Imai T, Sefat A S, Jin R, McGuire M A, Sales B C, and Mandrus D 2008 J. Phys.: Condens. Matter 20472201.

[28] Yildirim T Priprint arXiv:0807.3936. 